\title{
Odontogenic tumors: an 11-year international multicenter study
}

Felipe Martins Silveira ${ }^{a}$,, Carolina Carneiro Soares Macedo ${ }^{c}$, Carla Matos Vieira Borges ${ }^{\mathrm{a}}$, Matti Mauramo ${ }^{\mathrm{d}}$, Ana Carolina Uchoa Vasconcelos ${ }^{\mathrm{b}}$, Andresa Borges Soares ${ }^{a}$, Elizabeth Ferreira Martineza, Vera Cavalcanti de Araujo ${ }^{a}$, Marilena Vered ${ }^{e}$, Tuula Salo ${ }^{d}$, Fabricio Passador-Santos ${ }^{a}$

a Faculdade São Leopoldo Mandic, Instituto de Pesquisa São Leopoldo Mandic, Campinas, São Paulo, Brazil

${ }^{b}$ Diagnostic Centre for Oral Diseases, School of Dentistry, Federal University of Pelotas, Pelotas, Brazil

c Department of Microbiology, Immunobiology and Genetics, Center for Molecular Biology of the University of Vienna, Vienna, Austria

${ }^{d}$ Department of Oral and Maxillofacial Diseases, Clinicum, University of Helsinki, Helsinki, Finland e School of Dentistry, Faculty of Medicine, Tel Aviv University, Tel Aviv, Israel

\section{Corresponding author:}

Fabricio Passador-Santos

Department of Oral Pathology

São Leopoldo Mandic Research Institute

Campinas, São Paulo, Brazil

fabricio.passador-santos@slmandic.edu.br

Keywords: odontogenic tumors, epidemiology, World Health Organization 
Odontogenic tumors (OTs) are a heterogeneous group of lesions originating from the remnants of the tooth-forming apparatus (Nalabolu et al., 2016). The frequency rates of OTs vary from about $1 \%$ to $32 \%$ of all jawbone tumors (Adebayo et al., 2005; Sekerci et al., 2015).

OTs are divided into benign and malignant categories of unknown etiology (Sekerci et al., 2015; Wright and Tekkesin, 2017). The first internationally accepted classification of OTs was published by the World Health Organization (WHO) in 1971. It was then reviewed and updated in 1992, 2005, and in 2017 (Barnes et al., 2005; Sekerci et al., 2015; El-Naggar et al., 2017).

Several retrospective studies on OTs have been performed in different countries (Mosqueda-Taylor et al., 1997; Adebayo et al., 2005; Jing et al., 2007; Costa et al., 2012; Osterne et al., 2011), demonstrating variations in the clinical presentation of OTs. This multicenter study aimed to determine the prevalence and clinicopathologic presentation of OTs collected from three countries.

This retrospective study was approved by the Research Ethics Committee of the São Leopoldo Mandic Institute and Research Center (protocol number: 3.099.404). The pathology records of the following referral centers were evaluated over a 10-year period (1.1.2004-31-12.2014): Department of Oral Pathology, São Leopoldo Mandic Research Institute and Research Center (Brazil 1); Diagnostic Centre for Oral Diseases, School of Dentistry, Federal University of Pelotas (Brazil 2); Department of Oral and Maxillofacial Diseases, University of Helsinki (Finland); and School of Dentistry, Faculty of Medicine, Tel Aviv University (Israel). 
Cases of OTs were selected and classified according to the current WHO classification (2017) (Wright and Vered, 2017). The hematoxylin and eosin-stained sections were re-evaluated. Data regarding histopathological type, gender, age and anatomical site, were obtained from patient records.

The cases of OTs were divided according to the oral pathology center and its corresponding geographical areas. Based on the 10-year analysis, Brazil 1 was the center with the highest number of cases of OTs (437 cases), followed by Israel (163 cases), Finland (115 cases), and Brazil 2 (108 cases). The records of the two Brazilian centers were combined, resulting in 545 cases. A total of 823 cases of OTs were obtained (Figure 1).

Benign OTs (BOTs) were most common $(\mathrm{n}=814,98.9 \%)$. In all countries, the most frequent BOT was odontoma $(n=394,48.4 \%)$, followed by ameloblastoma $(n=253,31.0 \%)$. Odontogenic myxoma/myxofibroma ( $n$ $=66,8.1 \%$ ) was the third most common OT in Brazil and Israel, whereas in Finland, odontogenic myxoma, ameloblastic fibroma and odontogenic fibroma presented the same prevalence. Only 9 malignant OTs (MOTs) $(n=9,1.0 \%)$ were diagnosed in this series. Most cases were found in Israel (six out of nine), including five ameloblastic carcinomas and 1 clear cell odontogenic carcinoma. In Brazil, there was one case of ameloblastic fibrosarcoma and one case of ameloblastic carcinoma, similar to Finland (Tables 1 and 2).

OTs showed virtual equal distribution between males $(n=409,49.7 \%)$ and females $(n=407,49.4 \%)$. The mandible $(n=595,72.2 \%)$ was 2.9 times more affected than the maxilla $(n=199,24.1 \%)$, except for adenomatoid odontogenic tumor (Tables 1 and 2; Supplementary Table 1). The age of OTs patients ranged from 0 to 87 years, with a mean age of 22 years $(S D= \pm$ 
17.2). MOTs affected older patients when compared to BOTs, with a mean age of $55 \pm 22.6$ years.

In this multicenter study, the most common OT was odontoma, followed by ameloblastoma and odontogenic myxoma, similar to the data from previous publications (Mosqueda-Taylor et al., 1997; Tamme et al., 2004; Buchner et al., 2006; Chrysomali et al., 2013; Servato et al., 2013). However, it is difficult to compare these studies considering that each one is based on different classifications and study designs. It is worthwhile to mention that the prevalence of odontoma may have a bias. In several diagnostic services, the lesion is interpreted as a malformation or a hamartoma rather than a neoplasm, being discovered during routine radiographic examination and surgically treated only in cases with a secondary complication (Tamme et al., 2004).

This series of OTs showed a very similar frequency between males and females. There are studies in the literature reporting this similar incidence of OTs regarding gender (Ladeinde et al., 2005; Chrysomali et al., 2013;); however, some studies have reported a higher prevalence in males (Chrysomali et al., 2013; Nalabolu et al., 2016; Mascitti et al., 2019), while others also have shown females to be more affected (Osterne et al., 2011). There is no plausible explanation for these differences. In association with these results, this multicenter study showed that OTs affected mostly the mandible. The majority of the studies on OTs also demonstrated a strong predilection for the mandible (Sekerci et al., 2015; Mascitti et al., 2019; Rubini et al., 2017). Jaw-specific genetic mechanisms that regulate the evolution and development of upper and lower dentitions appear to differ and this may 
provide a partial explanation to the difference in the incidence of OTs in the mandible versus maxilla (Ferguson et al., 2000).

The present study showed that the average age of individuals affected by OTs was $22 \pm 17.2$ years. Different studies demonstrate that the average age varied, for example from 25.5 (Fernandes et al., 2005) to 46.7 years (Rubini et al., 2017). The variation may be related to the different samples and populations analyzed.

MOTs are extremely rare lesions, with similar reported incidences of 1.1\% (Mosqueda-Taylor et al., 1997), 1.18\% (Fernandes et al., 2005), and $1.26 \%$ (Adebayo et al., 2005). In this study, the malignant lesions were more common in males and in the mandible, affecting individuals of $55 \pm 21.6$ years. Curiously, there was a much higher prevalence of MOTs in the Israeli series. This could be attributed to the population genetic background or to specific characteristics of the pathology center where the lesions were diagnosed.

The present communication describes a panoramic view of the diagnosis of OTs from different continents, according to its most recent WHO classification. The study of the incidence of OTs in different geographic areas contributes to understand their trends over time. Besides that, this communication specially added the knowledge that the new WHO classification did not significantly impact the distribution of the most diagnosed OTs worldwide.

Conflicts of interests: none to declare.

\section{References}


Adebayo, E.T., Ajike, S.O., Adekeye, E.O. (2005) A review of 318 odontogenic tumors in Kaduna, Nigeria. J Oral Maxillofac Surg, 63(6), 811-9.

Barnes, L., Eveson, J., Reichart, P., Sidransky, D. WHO Head and Neck Tumours. Chapter 6: Odontogenic tumours. (3rd ed.), IARC: Lyon 2005, p. 283-327.

Buchner, A., Merrell, P.W., Carpenter, W.M. (2006) Relative frequency of central odontogenic tumors: a study of 1,088 cases from Northern California and comparison to studies from other parts of the world. $J$ Oral Maxillofac Surg, 64(9), 1343-52.

Chrysomali, E., Leventis, M., Titsinides, S., Kyriakopoulos, V., Sklavounou, A. (2013) Odontogenic tumors. J Craniofac Surg, 24(5), 1521-5.

Costa, D.O., Maurício, A.S., de-Faria, P.A., da-Silva, L.E., Mosqueda-Taylor, A., Lourenço, S.D. (2012) Odontogenic tumors: a retrospective study of four Brazilian diagnostic pathology centers. Med Oral Patol Oral Cir Bucal, 17(3), e389-94.

El-Naggar, Chan, J.K.C., Grandis, J.R., Takata, T., Slootweg, P. WHO classification of Head and Neck Tumours. Chapter 8: Odontogenic and maxilofacial bone tumours. (4th ed.), IARC: Lyon 2017, p.205-260.

Ferguson, C.A., Tucker, A.S., Sharpe, P.T. (2000) Temporospatial cell interactions regulating mandibular and maxillary arch patterning. Development, 127(2):403-12.

Fernandes, A.M., Duarte, E.C., Pimenta, F.J., Souza, L.N., Santos, V.R., Mesquita, R.A., de Aguiar, M.C. (2005) Odontogenic tumors: a study of 340 cases in a Brazilian population. J Oral Pathol Med, 34(10), 583-7. 
Jing, W., Xuan, M., Lin, Y., Wu, L., Liu, L., Zheng, X., Tang, W., Qiao, J., Tian, W. (2007) Odontogenic tumours: a retrospective study of 1642 cases in a Chinese population. Int J Oral Maxillofac Surg, 36(1):20-5.

Mascitti M, Togni L, Troiano G, Caponio VCA, Sabatucci A, Balercia A, Rubini C, Lo Muzio L, Santarelli A. Odontogenic tumours: a 25-year epidemiological study in the Marche region of Italy. Eur Arch Otorhinolaryngol. 2020 Feb;277(2):527-538.

Ladeinde, A.L., Ajayi, O.F., Ogunlewe, M.O., Adeyemo, W.L., Arotiba, G.T., Bamgbose, B.O., Akinwande, J.A. (2005) Odontogenic tumors: a review of 319 cases in a Nigerian teaching hospital. Oral Surg Oral Med Oral Pathol Oral Radiol Endod, 99(2), 191-5.

Mosqueda-Taylor, A., Ledesma-Montes, C., Caballero-Sandoval, S., PortillaRobertson, J., Ruíz-Godoy Rivera, L.M., Meneses-García, A. Odontogenic tumors in Mexico: a collaborative retrospective study of 349 cases. (1997) Oral Surg Oral Med Oral Pathol Oral Radiol Endod, 84(6), 672-5.

Nalabolu, G.R.K., Mohiddin, A., Hiremath, S.K.S., Manyam, R., Bharath, T.S., Raju, P.R. (2017). Epidemiological study of odontogenic tumours: An institutional experience. J Infect Public Health, 10(3), 324-330.

Osterne, R.L., Brito, R.G., Alves, A.P., Cavalcante, R.B., Sousa, F.B. (2011) Odontogenic tumors: a 5-year retrospective study in a Brazilian population and analysis of 3406 cases reported in the literature. Oral Surg Oral Med Oral Pathol Oral Radiol Endod, 111(4), 474-81.

Rubini, C., Mascitti, M., Santarelli, A., Tempesta, A., Limongelli, L., Favia, G., Maiorano, E. (2017) Odontogenic tumors: a retrospective clinicopathological study from two Italian centers. Pathologica, 109(1):35-46.

Sekerci, A.E., Nazlim, S., Etoz, M., Deniz, K., Yasa, Y. (2015) Odontogenic tumors: a collaborative study of 218 cases diagnosed over 12 years and 
comprehensive review of the literature. Med Oral Patol Oral Cir Bucal, 20(1), e34-44.

Servato, J.P., Prieto-Oliveira, P., de Faria, P.R., Loyola, A.M., Cardoso, S.V. (2013) Odontogenic tumours: 240 cases diagnosed over 31 years at a Brazilian university and a review of international literature. Int $J$ Oral Maxillofac Surg, 42(2), 288-93.

Tamme, T., Soots, M., Kulla, A., Karu, K., Hanstein, S.M., Sokk, A., Jõeste, E., Leibur, E. (2004) Odontogenic tumours, a collaborative retrospective study of 75 cases covering more than 25 years from Estonia. $J$ Craniomaxillofac Surg, 32(3), 161-5.

Wright, J.M., Tekkesin, M. (2017) Odontogenic tumors: where are we in 2017?. J Istanb Univ Fac Dent, 51(3 Suppl 1), S10-S30.

Wright, J.M., Vered, M. (2017). Update from the 4th Edition of the World Health Organization Classification of Head and Neck Tumours: Odontogenic and Maxillofacial Bone Tumors. Head Neck Pathol, 11(1), 68-77. 
Table 1. Frequency of odontogenic tumors by histological type and countries included in this multicenter study.

\begin{tabular}{|c|c|c|c|c|c|c|c|c|}
\hline \multirow[b]{3}{*}{ BENIGN ODONTOGENIC TUMORS } & \multicolumn{2}{|c|}{ Brazil } & \multicolumn{2}{|c|}{ Israel } & \multicolumn{2}{|c|}{ Finland } & \multicolumn{2}{|c|}{ Total } \\
\hline & $\mathrm{n}$ & $\%$ & $\mathrm{n}$ & $\%$ & $n$ & $\%$ & $\mathrm{n}$ & $\%$ \\
\hline & & & & & & & & \\
\hline Ameloblastoma & 175 & 32.2 & 49 & 31.2 & 29 & 25.4 & 253 & 31.0 \\
\hline Squamous odontogenic tumor & 3 & 0.5 & - & - & - & - & 3 & 0.4 \\
\hline Calcifying epithelial odontogenic tumor & 9 & 1.6 & 6 & 3.8 & - & - & 15 & 1.8 \\
\hline Adenomatoid odontogenic tumor & 21 & 3.9 & 2 & 1.3 & 1 & 0.9 & 24 & 2.9 \\
\hline Ameloblastic fibroma & - & - & 2 & 1.3 & 7 & 6.1 & 9 & 1.1 \\
\hline Primordial odontogenic tumor & - & - & - & - & - & - & - & - \\
\hline Odontoma & 275 & 50.6 & 61 & 38.8 & 58 & 50.9 & 394 & 48.4 \\
\hline Dentinogenic ghost cell tumor & - & - & - & - & - & - & - & - \\
\hline Odontogenic fibroma & 8 & 1.5 & 14 & 8.9 & 7 & 6.1 & 29 & 3.6 \\
\hline Odontogenic myxoma/myxofibroma & 40 & 7.4 & 19 & 12.2 & 7 & 6.1 & 66 & 8.1 \\
\hline Cementoblastoma & 12 & 2.2 & 4 & 2.5 & 5 & 4.4 & 21 & 2.6 \\
\hline Total benign odontogenic tumors & 543 & 66.7 & 157 & 19.3 & 114 & 14.0 & 814 & 98.9 \\
\hline \multicolumn{9}{|l|}{ MALIGNANT ODONTOGENIC TUMORS } \\
\hline Ameloblastic carcinoma & 1 & 50.0 & 5 & 83.3 & 1 & 100 & 7 & 77.8 \\
\hline Metastasizing (malignant) ameloblastoma & - & - & - & - & - & - & - & - \\
\hline Primary intraosseous carcinoma, NOS & - & - & - & - & - & - & - & - \\
\hline Sclerosing odontogenic carcinoma & - & - & - & - & - & - & - & - \\
\hline Clear cell odontogenic carcinoma & - & - & 1 & 16.6 & - & - & 1 & 11.1 \\
\hline Ghost cell odontogenic carcinoma & - & - & - & - & - & - & - & - \\
\hline Odontogenic carcinosarcoma & - & - & - & - & - & - & - & - \\
\hline Odontogenic sarcoma & 1 & 50.0 & - & - & - & - & 1 & 11.1 \\
\hline Total malignant odontogenic tumors & 2 & 22.2 & 6 & 66.6 & 1 & 11.1 & 9 & 1.0 \\
\hline Total odontogenic tumors & 545 & 66.2 & 163 & 19.8 & 115 & 13.9 & 823 & 100 \\
\hline
\end{tabular}

n, absolute frequency; \%, relative frequency; NOS, not otherwise specified; 
Table 2. Histological type, gender, age and anatomical site of the informed cases of odontogenic tumors from this multicenter study.

\begin{tabular}{|c|c|c|c|c|c|}
\hline & \multicolumn{2}{|c|}{ Total } & \multirow{2}{*}{$\begin{array}{l}\text { Gender } \\
\text { Male:Female }\end{array}$} & \multirow{2}{*}{$\begin{array}{l}\text { Age } \\
\text { Mean age } \pm \text { SD }\end{array}$} & \multirow{2}{*}{$\begin{array}{l}\text { Anatomic Location } \\
\text { Mandible:Maxilla }\end{array}$} \\
\hline & $\mathbf{n}$ & $\%$ & & & \\
\hline \multicolumn{6}{|l|}{ BENIGN ODONTOGENIC TUMORS } \\
\hline Odontoma & 394 & 48.4 & $1: 1$ & $16 \pm 12.8$ & $1.8: 1$ \\
\hline Ameloblastoma & 253 & 31 & $1.3: 1$ & $31.5 \pm 18.2$ & $23: 1$ \\
\hline Odontogenic Myxoma/Myxofibroma & 66 & 8.1 & $1: 1.5$ & $29 \pm 14.2$ & $1.5: 1$ \\
\hline Odontogenic Fibroma & 29 & 3.6 & $1: 1$ & $21 \pm 17.3$ & $2.7: 1$ \\
\hline Adenomatoid Odontogenic Tumor & 24 & 2.9 & $1: 2.6$ & $14 \pm 2.9$ & $1: 1.5$ \\
\hline Cementoblastoma & 21 & 2.6 & $1: 6.0$ & $26 \pm 17.0$ & 19:1 \\
\hline Calcifying Epithelial Odontogenic Tumor & 15 & 1.8 & $1: 2$ & $26 \pm 19.2$ & $3.6: 1$ \\
\hline Ameloblastic fibroma & 9 & 1.1 & $1: 1.25$ & $8.4 \pm 3.0$ & $2: 1$ \\
\hline Squamous Odontogenic Tumor & 3 & 0.4 & $1: 2$ & $39 \pm 19.9$ & $2: 1$ \\
\hline Total benign odontogenic tumors & 814 & 98.9 & $1: 1$ & $21 \pm 16.9$ & 2.9:1 \\
\hline \multicolumn{6}{|l|}{ MALIGNANT ODONTOGENIC TUMORS } \\
\hline Ameloblastic Carcinoma & 7 & 77.8 & $2.5: 1$ & $50.8 \pm 21.1$ & 1:0 \\
\hline Clear Cell Odontogenic Carcinoma & 1 & 11.1 & ND & 83 & Mandible \\
\hline Odontogenic Sarcoma & 1 & 11.1 & Female & 40 & ND \\
\hline Total malignant odontogenic tumors & 9 & 1.1 & $1.25: 1$ & $55 \pm 22.6$ & $1: 0$ \\
\hline Total odontogenic tumors & 823 & 100 & $1: 1$ & $22 \pm 17.2$ & 2.9:1 \\
\hline
\end{tabular}

$\mathbf{n}$, absolute frequency; \%, relative frequency; SD, standard deviation; ND, no data. 


\begin{tabular}{|c|c|c|c|c|c|c|c|c|c|c|c|c|c|}
\hline \multirow[b]{2}{*}{ BENIGN ODONTOGENIC TUMORS } & \multicolumn{2}{|c|}{ Male } & \multicolumn{2}{|c|}{ Female } & \multirow[b]{2}{*}{ M:F ratio } & \multirow[b]{2}{*}{ N.I. } & \multicolumn{2}{|c|}{ Mandible } & \multicolumn{2}{|c|}{ Maxilla } & \multirow[b]{2}{*}{ Man:Max ratio } & \multirow[b]{2}{*}{ N.I. } & \multirow[b]{2}{*}{ Total } \\
\hline & $n$ & $\%$ & $n$ & $\%$ & & & $n$ & $\%$ & $n$ & $\%$ & & & \\
\hline Ameloblastoma & 142 & 56.1 & 110 & 43.5 & $1.3: 1$ & 1 & 239 & 94.4 & 10 & 3.9 & 23.9:1 & 4 & 253 \\
\hline Squamous odontogenic tumor & 1 & 33.3 & 2 & 66.6 & 1:2 & 0 & 2 & 66.6 & 1 & 33.3 & $2: 1$ & 0 & 3 \\
\hline Calcifying epithelial odontogenic tumor & 5 & 33.3 & 10 & 66.6 & $1: 2$ & 0 & 11 & 73.3 & 3 & 20 & $3.6: 1$ & 1 & 15 \\
\hline Adenomatoid odontogenic tumor & 6 & 25.0 & 16 & 66.6 & 1:2.6 & 2 & 9 & 37.5 & 14 & 58.3 & $1: 1.5$ & 1 & 24 \\
\hline Ameloblastic fibroma & 4 & 44.4 & 5 & 55.5 & 1:1.25 & 0 & 6 & 66.6 & 3 & 33.3 & $2: 1$ & 0 & 9 \\
\hline Primordial odontogenic tumor & - & - & - & - & - & - & - & - & - & - & - & - & - \\
\hline Odontoma & 202 & 51.2 & 189 & 47.9 & $\sim 1: 1$ & 3 & 243 & 61.6 & 135 & 34.2 & $1.8: 1$ & 16 & 394 \\
\hline Dentinogenic ghost cell tumor & - & - & - & - & - & - & - & - & - & - & - & - & - \\
\hline Odontogenic fibroma & 15 & 51.7 & 14 & 48.2 & $\sim 1: 1$ & 0 & 19 & 65.5 & 7 & 24.1 & 2.7:1 & 2 & $29^{*}$ \\
\hline Odontogenic myxoma/myxofibroma & 26 & 39.4 & 39 & 59.0 & 1:1.5 & 1 & 39 & 59.0 & 25 & 37.9 & $1.5: 1$ & 2 & 66 \\
\hline Cementoblastoma & 3 & 14.2 & 18 & 85.7 & $1: 6$ & 0 & 19 & 90.5 & 1 & 4.7 & 19:1 & 1 & 21 \\
\hline Total benign odontogenic tumors & 404 & 49.6 & 403 & 49.5 & $\sim 1: 1$ & 7 & 587 & 72.1 & 199 & 24.4 & 2.9:1 & 27 & 814 \\
\hline \multicolumn{14}{|l|}{ MALIGNANT ODONTOGENIC TUMORS } \\
\hline Ameloblastic carcinoma & 5 & 71.4 & 2 & 28.5 & $2.5: 1$ & 0 & 7 & 100 & 0 & 0 & $1: 0$ & 0 & 7 \\
\hline Metastasizing (malignant) ameloblastoma & - & - & - & - & - & - & - & - & - & - & - & - & - \\
\hline Primary intraosseous carcinoma, NOS & - & - & - & - & - & - & - & - & - & - & - & - & - \\
\hline Sclerosing odontogenic carcinoma & - & - & - & - & - & - & - & - & - & - & - & - & - \\
\hline Clear cell odontogenic carcinoma & 0 & 0 & 1 & 25.0 & 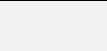 & 0 & 1 & 100 & 0 & 0 & $1: 0$ & 0 & 1 \\
\hline Ghost cell odontogenic carcinoma & - & - & - & - & - & - & - & - & - & - & - & - & - \\
\hline Odontogenic carcinosarcoma & - & - & - & - & - & - & - & - & - & - & - & - & - \\
\hline Odontogenic sarcoma & 0 & 0 & 1 & 25.0 & $0: 1$ & 0 & 0 & 0 & 0 & 0 & - & 1 & 1 \\
\hline Total malignant odontogenic tumors & 5 & 55.5 & 4 & 44.4 & $1.25: 1$ & 0 & 8 & 88.8 & 0 & 0 & 1:0 & 1 & 9 \\
\hline Total odontogenic tumors & 409 & 49.7 & 407 & 49.4 & $\sim 1: 1$ & 7 & 595 & 72.2 & 199 & 24.1 & 2.9:1 & 28 & 823 \\
\hline
\end{tabular}

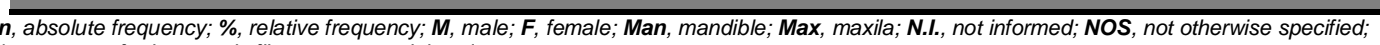

" one case of odontogenic fibroma was peripheral 
Figure 1. Flowchart showing the sample selection of the multicenter study

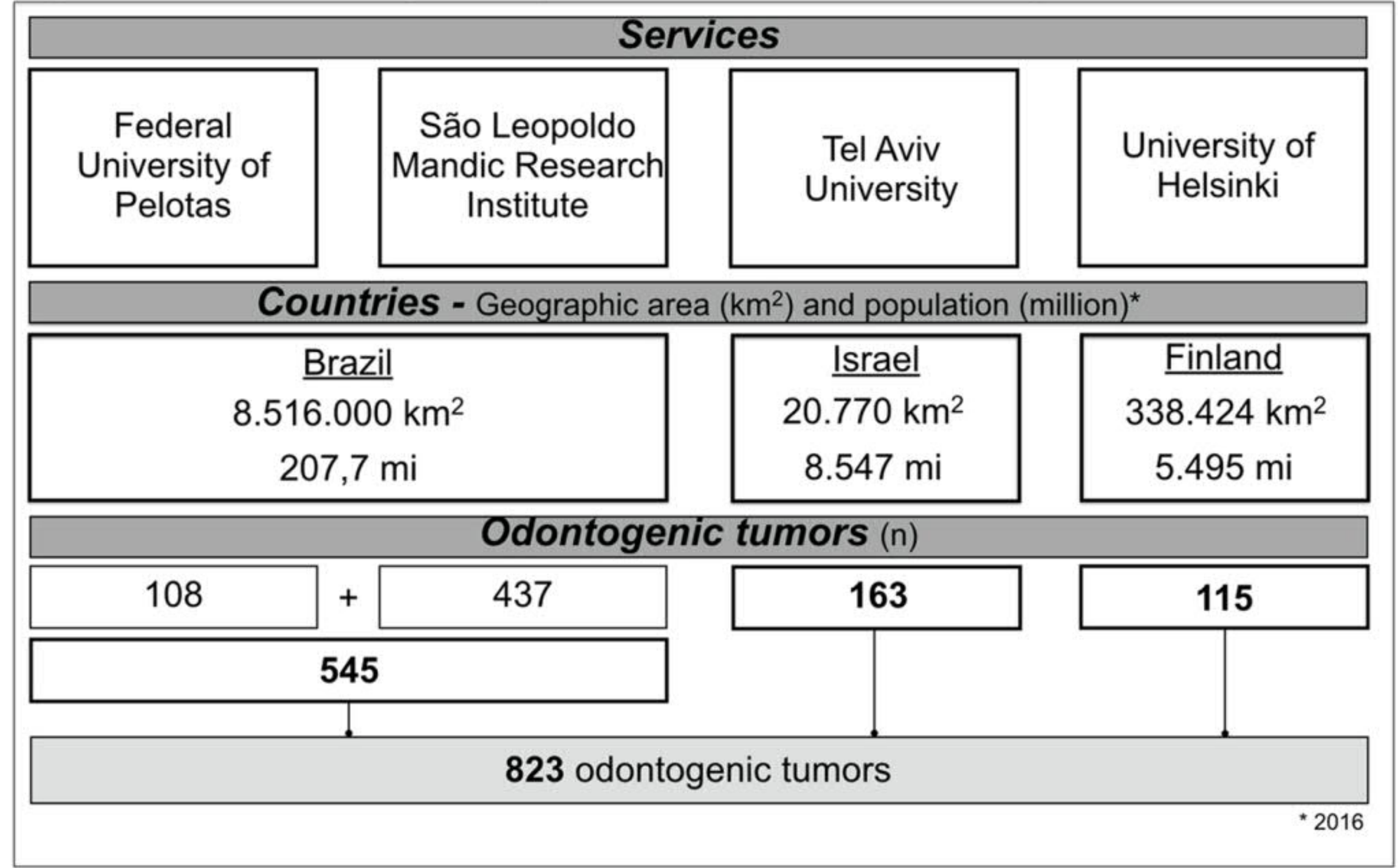

